\title{
OBSTRUCTION THEORY FOR SUPERMANIFOLDS AND DEFORMATIONS OF SUPERCONFORMAL STRUCTURES
}

\author{
KOWSHIK BETTADAPURA
}

(Received 9 November 2016; first published online 15 March 2017)

2010 Mathematics subject classification: primary 32C11; secondary 14D21, 14H15, 14H81, 32G05, $58 \mathrm{~A} 50$.

Keywords and phrases: complex supermanifold, super Riemann surface, obstruction, deformation, splitting.

In this thesis we study two related topics: (1) foundations of complex supermanifolds and (2) deformation theory for $\mathcal{N}=1$ and $\mathcal{N}=2$ super Riemann surfaces. Each topic is summarised below.

\section{Complex supermanifolds}

It is in theories of physics with supersymmetry where one might first encounter the notion of a supermanifold and in superstring theory one finds the notion of a complex (and complex-smooth) supermanifold. Chapters 2 to 5 in this thesis treat obstruction theory for complex supermanifolds in two broad settings:

(i) obstructions to extending a thickening of a given order to a supermanifold; and

(ii) obstructions to splitting a given supermanifold.

Regarding (i), this problem was first studied by Eastwood and LeBrun in [3], where the space of obstructions to extending a thickening of a given order was identified. We present in this thesis another proof of this result using classical methods of deformation theory for compact, complex manifolds in [4]. As a result of our proof, necessary and sufficient conditions for the existence of an obstructed thickening are deduced. This leads naturally to a certain decomposition of the space of thickenings of a given order into: (1) obstructed thickenings; (2) pseudo-supermanifolds and (3) supermanifolds. In Chapter 4, the existence of an obstructed thickening is inferred on the complex projective plane equipped with a rank-3, split vector bundle and a rank-3, nonsplit, decomposable vector bundle.

Thesis submitted to the Australian National University in May 2016; degree approved on 9 November 2016; supervisor Peter Bouwknegt.

(C) 2017 Australian Mathematical Publishing Association Inc. 0004-9727/2017 \$16.00 
In the second setting (ii), we consider obstruction theory related to splitting. A supermanifold $\mathfrak{X}$ is said to be split if it is isomorphic to its split model, which is a space equipped with a $\mathbb{Z}$-graded, $\mathbb{Z}_{2}$-commutative sheaf of rings. The question of whether a given supermanifold is split is central to the study of obstruction theory here and the obstruction classes to splitting, which are classes contrived to measure the failure for a given supermanifold to be split, are described in considerable detail. The main result derived here is the description of these classes via a certain vector field, called the grading vector field, which lives on the split model. It is shown that this vector field will lift to an obstruction class to splitting, whenever this class exists. This observation is then used to give a characterisation of the Atiyah class of a complex supermanifold. As a consequence of these relations, we deduce a result, first obtained by Koszul in [5], which is referred to as the Koszul splitting theorem. It asserts: if a supermanifold $\mathfrak{X}$ admits a global, holomorphic connection on its tangent bundle, then it is split. The proof of the Koszul splitting theorem presented in this thesis is quite different to that given by Koszul in [5] and is motivated by the results on Atiyah classes obtained by Donagi and Witten in [1]. As such, the proof given here can be insightful in its own right. For instance, in the proof presented in this thesis, the role played by the higher obstruction classes is quite clear.

\section{Deformations of superconformal structures}

In superstring theory, $\mathcal{N}=1$ super Riemann surfaces are the natural objects of interest whose moduli one wishes to study. The moduli space of a genus- $g$, super Riemann surface $\mathfrak{M}_{g}$ is an object in supergeometry (that is, the supermanifold analogue of a stack or orbifold) and its obstruction theory has been a topic of revived interest. In two recent papers of Donagi and Witten $[1,2]$, it was shown that $\mathfrak{M}_{g}$ and $\mathfrak{M}_{g, 1}$ is nonsplit (by virtue of the stronger property of it being nonprojected) for genus $g \geq 5$. In this thesis we study aspects of the objects parametrised by $\mathfrak{M}_{g}$ which are $\mathcal{N}=1$ super Riemann surfaces and a related construct of $\mathcal{N}=2$ super Riemann surfaces.

An $\mathcal{N}=1$ super Riemann surface $\mathcal{S}$ is the primary object of interest in superstring theory. A deformation of $\mathcal{S}$ is, first and foremost, a complex supermanifold of some dimension and, in Chapter 6, we study the obstruction theory of these deformations. More precisely, the Kodaira-Spencer map of a deformation over $\mathbb{C}^{0 \mid 1}$ is related to the obstruction class to splitting the deformation. This result is known in the literature and we present a generalisation of it to odd deformations of second order, which are deformations over $\mathbb{C}^{0 \mid 2}$. Based on our study of the obstruction theory of odd, secondorder deformations of $\mathcal{S}$, we arrive at a natural question regarding the characterisation of deformations of any order.

Regarding the $\mathcal{N}=2$ setting, we begin by giving a general definition of a superconformal structure on a supermanifold of any dimension, after which we specialise to rank-2 vector bundles on complex curves in Chapter 7. If a (1|2)dimensional supermanifold $\mathfrak{X}$ over a Riemann surface admits a superconformal structure, then $\mathfrak{X}$ is referred to as an $\mathcal{N}=2$ super Riemann surface. Denote by 
$\mathfrak{M}_{g}^{\mathcal{N}=2}$ the moduli space of $\mathcal{N}=2$ super Riemann surfaces. In this chapter we investigate a relation from the literature which asserts: there exists an embedding of supermoduli spaces $\mathfrak{M}_{g} \subset \mathfrak{M}_{g}^{\mathcal{N}=2}$. We prove this assertion at the level of odd, infinitesimal deformations. Then, based on this result and the compatibility lemma of Donagi and Witten in [2], we conclude this chapter with sketches of an argument as to why $\mathfrak{M}_{g}^{\mathcal{N}=2}$ must be nonsplit, just like its $\mathcal{N}=1$ counterpart.

\section{References}

[1] R. Donagi and E. Witten, 'Super Atiyah classes and obstructions to splitting of supermoduli space', Preprint, 2014, arXiv:1404.6257 [hep-th].

[2] R. Donagi and E. Witten, 'Supermoduli space is not projected', String-Math 2012, Proc. Symp. Pure Math. 90 (2015), 19-72.

[3] M. Eastwood and C. LeBrun, 'Thickening and supersymmetric extensions of complex manifolds', Amer. J. Math. 108(5) (1986), 1177-1192.

[4] K. Kodaira, Complex Manifolds and Deformation of Complex Structures (Springer, New York, 1986).

[5] J. Koszul, 'Connections and splittings of supermanifolds', Differential Geom. Appl. 4 (1994), $151-161$.

KOWSHIK BETTADAPURA, Mathematical Sciences Institute, Australian National University, Canberra, ACT 2601, Australia e-mail: kowshik.bettadapura@anu.edu.au 